Artículo

\title{
Parámetros genéticos y aptitud combinatoria de líneas de maíz para grano
}

\author{
Gilberto Rodríguez Pérez ${ }^{1}$ \\ José Elías Treviño Ramírez ${ }^{2}$ \\ Ma. del Carmen Ojeda Zacarias ${ }^{2}$ \\ Francisco Cervantes Ortiz ${ }^{3}$ \\ Miguel Angel Avila Perches ${ }^{4}$ \\ Alfredo Josué Gámez Vázquez ${ }^{4 \S}$ \\ ${ }^{1}$ Tecnológico Nacional de México-Campus Valle del Yaqui. Avenida Tecnológico Block 611, Valle del \\ Yaqui, Bácum, Ciudad Obregón, Sonora. CP. 85276. Tel. 643 4357100. (grodriguez263@ hotmail.com). \\ ${ }^{2}$ Facultad de Agronomía-Universidad Autónoma de Nuevo León. Carretera Zuazua-Marín km 17.5, Marín, \\ Nuevo León. (eliastrevino_ramirez@hotmail.com; ojeda.cz@ hotmail.com. ${ }^{3}$ Tecnológico Nacional de \\ México-Campus Roque. Carretera Celaya-Juventino Rosas km 8, Celaya, Guanajuato. CP. 38110. Tel. \\ 461616263, ext. 135. (frcervantes@itroque.edu.mx). ${ }^{4}$ Campo Experimental Bajío-INIFAP. Carretera \\ Celaya-San Miguel de Allende km 6.5, Celaya, Guanajuato. CP. 38110. (avila.miguel@inifap.gob.mx). Tel. \\ 8000882222 , ext. 85228.
}

Autor para correspondencia: gamez.josue@inifap.gob.mx.

\section{Resumen}

La aptitud combinatoria general (ACG) y la específica (ACE) se usan para identificar combinaciones híbridas entre progenitores con rendimiento mayor o menor que el comportamiento promedio esperado de las líneas progenitoras. El objetivo de este estudio fue evaluar la ACG de 7 líneas como hembras (H) y 4 líneas macho (M); así como la ACE de las 28 cruzas realizadas; como hipótesis se planteó, que los cruzamientos entre líneas con características contrastantes producirán híbridos sobresalientes. La obtención de las líneas con un nivel de endogamia de S3, los cruzamientos y evaluación de estos últimos se realizó en el Campo Experimental de la Facultad de Agronomía de la Universidad Autónoma de Nuevo León, México, las cruzas simples se evaluaron en un diseño experimental de bloques completos al azar con tres repeticiones. El análisis genético se realizó con el diseño II de apareamiento de Carolina del Norte de Comstock y Robinson. Los progenitores, que presentaron más altos rendimientos fueron M2 $\left(8.12 \mathrm{t} \mathrm{ha}^{-1}\right)$ y M4 $\left(7.9 \mathrm{t} \mathrm{ha}^{-1}\right)$ considerados como machos y como hembras $\mathrm{H} 3$ y H5 $\left(8.13 \mathrm{t} \mathrm{ha}^{-1}\right)$ ambas con mismo rendimiento; sin embargo, los mayores efectos de ACG fueron en M2 (0.19), M1(0.08), H4 (0.14) y H2 (0.13) en rendimiento de grano. Los efectos mayores en ACE se observaron en las cruzas H4xM2 (1.17 t $\left.\mathrm{ha}^{-1}\right)$, H6xM3 (0.84 t ha- $)$ y H2xM3 $\left(0.58 \mathrm{t} \mathrm{ha}^{-1}\right)$. En los parámetros genéticos para rendimiento de grano, la varianza ambiental superó a la varianza aditiva y de dominancia, la heredabilidad en sentido amplio y estrecho fueron 87.14 y $52.15 \%$, respectivamente.

Palabras clave: capacidad combinatoria, cruzas, líneas endogámicas, machos y hembras.

Recibido: agosto de 2020

Aceptado: octubre de 2020 


\section{Introducción}

Los programas de mejoramiento genético dedicados a la formación de híbridos y variedades comerciales de maíz requieren generar líneas con potencial de rendimiento alto, comportamiento agronómico y aptitud combinatoria. Las líneas que reúnen estas características presentan resultados satisfactorios en combinaciones híbridas (Fan et al., 2008). Los conceptos de aptitud combinatoria general (ACG) y específica (ACE) permiten expresar el comportamiento promedio de una línea en sus combinaciones híbridas y designar las combinaciones híbridas que resulten superiores, o no, a las esperadas en relación con el promedio de la ACG de las dos líneas progenitoras. Estas se usan con frecuencia para estimar efectos y varianzas de ACG y ACE (Sprague y Tatum, 1942).

Un propósito importante del mejoramiento genético de maíz por hibridación es generar cruzas que superen en rendimiento de grano a las variedades criollas, mejoradas e híbridos comerciales. Si en una población los efectos de aptitud combinatoria general son más importantes que los efectos específicos, es recomendable mejorar a la población por selección recurrente; por el contrario, si los efectos de aptitud combinatoria específica son los más importantes, la población deberá mejorarse por hibridación (Reyes et al., 2004).

El conocimiento de la acción génica que controla los caracteres de interés económico es básico para la planeación de un programa de mejoramiento genético mediante la aptitud combinatoria de los progenitores, el mejorador obtiene mayores logros en su programa de mejoramiento porque permite seleccionar progenitores con un comportamiento promedio aceptable en una serie de cruzamientos e identificar combinaciones específicas con un comportamiento superior a lo esperado, con base en el promedio de los progenitores (De la Cruz et al., 2005; Guillén et al., 2009).

Gran parte de la diversidad genética del maíz nativo de México aún se puede encontrar en los campos agrícolas en forma de variedades criollas, ya que sólo en $23 \%$ de la superficie sembrada con maíz se usa semilla mejorada (Polanco, 2008). Además, los agricultores han identificado características favorables a sus necesidades y preferencias, por lo que se requiere buscar nuevas combinaciones genéticas creativas para generar mayor variación (Louette y Smale, 1996).

El objetivo de este estudio fue identificar una alternativa que mejore el rendimiento de los híbridos, generados del cruzamiento entre líneas endogámicas derivadas de los grupos Pinto Amarillo y Liebre recolectadas en el centro-norte de Nuevo León, México. La hipótesis fue que los cruzamientos entre progenitores con características contrastantes provenientes de las poblaciones Pinto Amarillo y Liebre producirán híbridos con altos niveles de heterosis para rendimiento de grano y sus componentes.

\section{Materiales y métodos}

El estudio se realizó bajo condiciones de riego en el Campo Experimental de la Facultad de Agronomía, de la Universidad Autónoma de Nuevo León, ubicado en el km 17.5 de la carretera Zuazua-Marín, Marín, Nuevo León, México, que se localiza en $25^{\circ} 53^{\prime}$ latitud norte, $100^{\circ} 03^{\prime}$ longitud oeste, con una altitud de $375 \mathrm{~m}$, temperatura promedio anual de $22{ }^{\circ} \mathrm{C}$ y precipitación anual promedio de $573 \mathrm{~mm}$ (INEGI, 2013). Se utilizaron 11 líneas (Cuadro 1) con un nivel de endogamia de S3, las cuales fueron cruzadas en un diseño II de Carolina del Norte, se usaron 7 progenitores como hembra $(\mathrm{H})$ y cuatro como macho, por lo que se generaron 28 cruzas simples. 
Cuadro 1. Origen y descripción de las líneas de maíz con el nivel de endogamia $S_{3}$ participantes como progenitores en la formación de 28 híbridos.

\begin{tabular}{ccl}
\hline Línea & Municipio & Formación y características de la línea \\
\hline H-1 & Los Ramones & Población 24 de Pinto Amarillo. Precoz tolerante a sequía \\
H-2 & Mina & Población 13 de Pinto Amarillo. Intermedio de amplia adaptabilidad \\
H-3 & Parás & Población 71 de Pinto Amarillo. Intermedio tolerante a sequía \\
H-4 & Pesquería & Población 11 de Pinto Amarillo. Intermedio de amplia adaptabilidad \\
H-5 & China & Población 50 de Pinto Amarillo. Intermedio tolerante a sequía \\
H-6 & García & Población 46 de Pinto Amarillo. Intermedia de amplia adaptabilidad \\
H-7 & Bustamante & Población 16 de Pinto Amarillo. Precoz tolerante a sequía \\
M-1 & Pesquería & Población 10 de Liebre. Intermedio de amplia adaptabilidad \\
M-2 & Los Ramones & Población 19 de Liebre. Precoz tolerante a sequía \\
M-3 & Los Ramones & Población 37 de Liebre. precoz tolerante a sequía \\
M-4 & Cadereyta & Población 63 de Liebre. precoz de amplia adaptabilidad \\
\hline
\end{tabular}

Las líneas de Pinto Amarillo presentan rendimientos aceptables (Cuadro 2) y grano semidentado amarillo, las líneas de Liebre son precoces, tolerantes a déficit hídrico y de grano blanco semicristalino y tolerantes a estrés hídrico. Las 11 líneas fueron seleccionadas con base en resultados de un análisis de conglomerados (Rodríguez et al., 2012). Se sembraron seis surcos de cada línea endogámica progenitora (Cuadro 1) y se utilizaron 35 plantas para realizar los cruzamientos entre hembras y machos.

Los 28 híbridos se evaluaron en un diseño de bloques completos al azar con tres repeticiones, en una parcela experimental de 4 surcos de $5 \mathrm{~m}$ de largo y $0.8 \mathrm{~m}$ de ancho, con siete plantas por metro lineal para obtener una densidad de 87500 plantas ha ${ }^{-1}$. Las variables estudiadas fueron: altura de planta (AP), altura de mazorca (AM), días a floración femenina (FF), longitud de mazorca (LM), diámetro de mazorca (DM), diámetro de olote (DO), número de hileras por mazorca (HM), número de granos por hilera $(\mathrm{GH})$ y rendimiento de grano $(\mathrm{RG})$. Todas las plantas de la parcela se cosecharon y se pesó el grano para transformar el dato en $\mathrm{t} \mathrm{ha}^{-1}$, ajustándose a $13 \%$ de humedad.

El análisis genético se hizo con el diseño II de apareamiento de Carolina del Norte de Comstock y Robinson (1948), cuyo modelo lineal fue el siguiente: $\mathrm{Y}_{\mathrm{ijk}}=\mu+\mathrm{M}_{\mathrm{i}}+\mathrm{H}_{\mathrm{j}}+\phi_{\mathrm{ij}}+\varepsilon_{\mathrm{ijk}}$. Donde: $\mathrm{i}=1$. $2 \mathrm{~m}$ (machos); j=1. $2 \mathrm{~h}$ (hembras); $\mathrm{k}=1.2 \mathrm{r}$ (rep); $\mathrm{Y}_{\mathrm{ijk}}=$ observación de la cruza entre el iésimo macho y la j-ésima hembra en la k-ésima repetición; $\mu=$ media general; $\mathrm{M}_{\mathrm{i}}$ y $\mathrm{H}_{\mathrm{j}}=$ efecto del i-ésimo macho y jésima hembra; $\phi_{\mathrm{ij}}=$ efecto de la interacción del i-ésimo macho con la j-ésima hembra; $\varepsilon_{\mathrm{ijk}}=$ error experimental.

La estimación de los efectos de aptitud combinatoria general (ACG) para los machos y hembras y de aptitud combinatoria específica (ACE) para las cruzas, se hizo según la propuesta de Sprague y Tatum (1942): $g_{i}=\widetilde{Y}_{i .}-\widetilde{Y}_{. .} \cdot g_{j}=\widetilde{Y}_{. j}-\widetilde{Y}_{. .} . S_{i j}=Y_{i j}-g_{i}-g_{j}-\widetilde{Y}_{. .}$Donde: $g_{i} \cdot g_{j}$ y $S_{i j}$ son los efectos de ACG

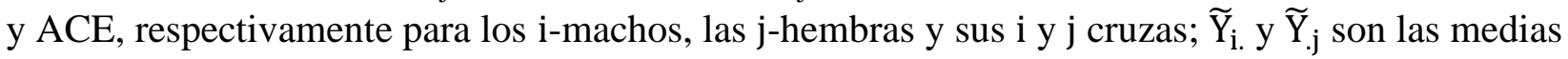
de los machos y las hembras; $\mathrm{Y}_{\mathrm{ij}}$ es el valor de la cruza $\mathrm{i} * \mathrm{j}$ y $\widetilde{\mathrm{Y}}_{. .}$es la media de las $\mathrm{i} * \mathrm{j}$ cruzas. 
La diferencia estadística entre las ACG de los progenitores machos y hembras y de las ACE de las cruzas, se determinó mediante la diferencia mínima significativa, (DMS, 0.05), $\alpha=\mathrm{EE}$ x t ( $\alpha 2-1$. gl ee), donde $\mathrm{EE}=$ error estándar en la comparación de medias; $\mathrm{EE}=\sqrt{2 \mathrm{CME}}(\mathrm{RM})-1 ; \mathrm{R}=$ repeticiones; $\mathrm{M}=$ machos; $\mathrm{H}=$ hembras; $\mathrm{gl}=$ grados de libertad; $\mathrm{y}$ ee = error experimental.

Los componentes genéticos se estimaron de acuerdo a valores de las esperanzas de los cuadrados medios del análisis de varianza; varianza de machos $\left(\delta^{2} \mathrm{M}=\mathrm{M} 4-\mathrm{m} 2 / \mathrm{rH}\right)$, varianza de hembras $\left(\delta^{2} \mathrm{H}=\mathrm{M} 3-\mathrm{M} 2 / \mathrm{rM}\right)$, varianza machos $\mathrm{x}$ hembras $\left(\delta^{2} \mathrm{MH}=\mathrm{M} 2-\mathrm{M} 1 / \mathrm{r}\right)$, varianza fenotípica $\left(\delta^{2} \mathrm{~F}=\right.$ $\left.\delta^{2} \mathrm{M}+\delta^{2} \mathrm{H}+\delta^{2} \mathrm{MH}+\delta^{2} \mathrm{e}\right)$, varianza aditiva $\left(\delta^{2} \mathrm{~A}=4 \delta^{2} \mathrm{M}\right)$, varianza de dominancia $\left(\delta^{2} \mathrm{D}=4\left[\delta^{2} \mathrm{H}-\right.\right.$ $\left.\delta^{2} \mathrm{M}\right]$, heredabilidad en sentido estrecho $\left(\mathrm{h}^{2}=\delta^{2} \mathrm{~A} / \delta^{2} \mathrm{~F}\right)$ y heredabilidad en sentido amplio $\left(\mathrm{H}^{2}=\right.$ $\left.\delta^{2} \mathrm{G} / \delta^{2} \mathrm{~F}\right)$.

\section{Resultados y discusión}

Existieron diferencias estadísticas (Cuadro 2) entre híbridos, para rendimiento de grano (RG), altura de mazorca (AM), floración femenina (FF), longitud de mazorca (LM) y granos por hilera $(\mathrm{GH})$; para machos, en altura de mazorca (AM), floración femenina (FF), longitud de mazorca (LM), diámetro de olote (DO) y granos por hilera $(\mathrm{GH})$, en hembras, para rendimiento de grano (RG), altura de mazorca (AM) y longitud de mazorca (LM); en la interacción MxH fue significativa en RG, AM, FF, LM, DO y GH.

Cuadro 2. Cuadrados medios del análisis de varianza y nivel de significancia estadística del análisis del diseño II de Carolina de Norte. Marín, Nuevo León.

\begin{tabular}{lllllllllll}
\hline \multicolumn{1}{c}{ FV } & GL & RG & AP & AM & FF & LM & DM & DO & HM & GH \\
\hline Repeticiones & 2 & 0.5 & 0.06 & 0.01 & 10.08 & 1.16 & 0.16 & 0.13 & 0.68 & 14.89 \\
Híbridos & 27 & $0.74^{*}$ & $0.81 \mathrm{~ns}$ & $0.14^{*}$ & $217.38^{*}$ & $9.16^{*}$ & $0.15 \mathrm{~ns}$ & $0.7 \mathrm{~ns}$ & $2.25 \mathrm{~ns}$ & $61.36^{*}$ \\
Machos & 3 & $1.1 \mathrm{~ns}$ & $0.02 \mathrm{~ns}$ & $0.22^{*}$ & $220.57^{*}$ & $8.47^{*}$ & $0.09 \mathrm{~ns}$ & $0.23^{*}$ & $1.3 \mathrm{~ns}$ & $58.01^{*}$ \\
Hembras & 6 & $1.19^{*}$ & $0.03 \mathrm{~ns}$ & $0.02^{*}$ & $5.75 \mathrm{~ns}$ & $5.01^{* *}$ & $0.09 \mathrm{~ns}$ & $0.03 \mathrm{~ns}$ & $0.19 \mathrm{~ns}$ & $13.06 \mathrm{~ns}$ \\
$\mathrm{M} * \mathrm{H}$ & 18 & $0.98^{*}$ & $0.06 \mathrm{~ns}$ & $0.03^{*}$ & $25.59^{*}$ & $5.31^{*}$ & $0.11 \mathrm{~ns}$ & $0.04^{*}$ & $0.79 \mathrm{~ns}$ & $22.34^{*}$ \\
Error & 54 & 0.82 & 0.04 & 0.02 & 13.53 & 1.69 & 0.21 & 0.07 & 1.95 & 14.95 \\
Total & 83 & 0.88 & 0.04 & 0.03 & 94.61 & 2.95 & 0.17 & 0.07 & 1.52 & 17.97 \\
CV $(\%)$ & & 11.55 & 10.45 & 9.48 & 4.84 & 9.37 & 10.80 & 5.49 & 10.74 & 11.8 \\
\hline
\end{tabular}

$\mathrm{RG}=$ rendimiento de grano $\mathrm{AP}=$ altura de planta; $\mathrm{AM}=$ altura de mazorca; $\mathrm{FF}=$ floración femenina; $\mathrm{LM}=$ longitud de mazorca; $\mathrm{DM}=$ diámetro de mazorca; $\mathrm{DO}=$ diámetro de olote; $\mathrm{HM}=$ hileras por mazorca; $\mathrm{GH}=$ granos por hilera.

Estas diferencias se deben al origen contrastante del material genético estudiado, lo que confirma los resultados obtenidos por De la Cruz et al. (2005); De la Rosa et al. (2006); Borghi et al. (2012) quienes identificaron híbridos, machos y hembras en genotipos de maíz con potencial de rendimiento y sus componentes agronómicos como resultado de la variabilidad genética alta entre poblaciones de las que se derivaron las líneas y por la diversidad genética entre las líneas.

Los coeficientes de variación usados como una medida de precisión en la conducción de los experimentos (Kang et al., 1999), para rendimiento de grano, fue de $11.55 \%$, considerado como aceptable, los coeficientes en los componentes del rendimiento fluctuaron entre 4.84 y $11.8 \%$, debido a que son caracteres de menor variación. 


\section{Comportamiento del rendimiento y sus componentes}

En el Cuadro 3 las hembras $\mathrm{H} 3$ y $\mathrm{H} 5$ presentaron en promedio rendimiento de grano con $8.13 \mathrm{t}$ ha $^{-1}$; sin embargo, H6, H7, H2 y H1 estuvieron en el segundo grupo estadístico con promedios de $8.01,7.88,7.84$ y $7.54 \mathrm{t} \mathrm{ha}^{-1}$ respectivamente; el rendimiento sobresaliente en las hembras pudo deberse a su ciclo intermedio y tolerancia a sequía, en los machos sobresalieron M2 (8.12 $\mathrm{t} \mathrm{ha}^{-1}$ ) y M4 (7.9 $\left.\mathrm{t} \mathrm{ha}^{-1}\right)$. Es probable que la combinación entre machos y hembras influyera en el comportamiento de las cruzas, ya que la similitud en el rendimiento obtenido de las líneas macho se debe a que son de ciclo intermedio, lo que se refleja en sus rendimientos superiores al resto de los machos. Wong et al. (2007) también relacionaron este componente lo que permite una alta expresión con un mayor rendimiento. Por tanto, se esperaría un buen comportamiento al combinar estos progenitores en la formación de híbridos (Soengas et al., 2003).

Cuadro 3. Promedios del rendimiento y componentes agronómicos, de las cruzas simples agrupadas por progenitor hembra y macho. Marín, Nuevo León.

\begin{tabular}{lccccccccc}
\hline Progenitor & $\begin{array}{c}\text { RG } \\
\left(\mathrm{t} \mathrm{ha}^{-1}\right)\end{array}$ & $\begin{array}{c}\text { AP } \\
(\mathrm{m})\end{array}$ & $\begin{array}{c}\text { AM } \\
(\mathrm{m})\end{array}$ & $\begin{array}{c}\text { FF } \\
(\text { días })\end{array}$ & $\begin{array}{c}\text { LM } \\
(\mathrm{cm})\end{array}$ & $\begin{array}{c}\text { DM } \\
(\mathrm{cm})\end{array}$ & $\begin{array}{c}\text { DO } \\
(\mathrm{cm})\end{array}$ & HM & GH \\
\hline H1 & $7.54 \mathrm{ab}$ & $1.96 \mathrm{a}$ & $1.32 \mathrm{~b}$ & $76 \mathrm{a}$ & $12.97 \mathrm{~d}$ & $4.36 \mathrm{a}$ & $2.48 \mathrm{a}$ & $13.16 \mathrm{a}$ & $31.66 \mathrm{a}$ \\
$\mathrm{H} 2$ & $7.84 \mathrm{ab}$ & $1.99 \mathrm{a}$ & $1.39 \mathrm{ab}$ & $76.58 \mathrm{a}$ & $13.14 \mathrm{~cd}$ & $4.15 \mathrm{a}$ & $2.37 \mathrm{a}$ & $13.08 \mathrm{a}$ & $31.75 \mathrm{a}$ \\
$\mathrm{H} 3$ & $8.13 \mathrm{a}$ & $1.97 \mathrm{a}$ & $1.44 \mathrm{a}$ & $76.5 \mathrm{a}$ & $14.01 \mathrm{abcd}$ & $4.23 \mathrm{a}$ & $2.51 \mathrm{a}$ & $13 \mathrm{a}$ & $33.33 \mathrm{a}$ \\
H4 & $7.29 \mathrm{~b}$ & $1.95 \mathrm{a}$ & $1.43 \mathrm{a}$ & $76.25 \mathrm{a}$ & $13.72 \mathrm{bcd}$ & $4.1 \mathrm{a}$ & $2.4 \mathrm{a}$ & $13 \mathrm{a}$ & $32 \mathrm{a}$ \\
H5 & $8.13 \mathrm{a}$ & $1.93 \mathrm{a}$ & $1.4 \mathrm{ab}$ & $75.5 \mathrm{a}$ & $14.31 \mathrm{ab}$ & $4.21 \mathrm{a}$ & $2.38 \mathrm{a}$ & $13 \mathrm{a}$ & $32.91 \mathrm{a}$ \\
H6 & $8.01 \mathrm{ab}$ & $2.05 \mathrm{a}$ & $1.39 \mathrm{ab}$ & $75.75 \mathrm{a}$ & $14.05 \mathrm{abc}$ & $4.31 \mathrm{a}$ & $2.4 \mathrm{a}$ & $13 \mathrm{a}$ & $34.58 \mathrm{a}$ \\
H7 & $7.88 \mathrm{ab}$ & $1.86 \mathrm{a}$ & $1.38 \mathrm{ab}$ & $74.58 \mathrm{a}$ & $14.82 \mathrm{a}$ & $4.23 \mathrm{a}$ & $2.4 \mathrm{a}$ & $12.75 \mathrm{a}$ & $33 \mathrm{a}$ \\
DMS (0.05) & 0.74 & 0.17 & 0.11 & 3.01 & 1.06 & 0.37 & 0.21 & 12.36 & 33.21 \\
M1 & $7.75 \mathrm{a}$ & $1.97 \mathrm{a}$ & $1.29 \mathrm{c}$ & $81.14 \mathrm{a}$ & $14.39 \mathrm{a}$ & $4.31 \mathrm{a}$ & $2.58 \mathrm{a}$ & $12.95 \mathrm{a}$ & $33 \mathrm{a}$ \\
M2 & $8.1 \mathrm{a}$ & $1.95 \mathrm{a}$ & $1.36 \mathrm{bc}$ & $81.19 \mathrm{a}$ & $13.22 \mathrm{~b}$ & $4.21 \mathrm{a}$ & $2.35 \mathrm{~b}$ & $12.66 \mathrm{a}$ & $30.38 \mathrm{~b}$ \\
M3 & $7.58 \mathrm{a}$ & $1.91 \mathrm{a}$ & $1.39 \mathrm{~b}$ & $80.67 \mathrm{a}$ & $14.42 \mathrm{a}$ & $4.15 \mathrm{a}$ & $2.36 \mathrm{~b}$ & $13.19 \mathrm{a}$ & $34.24 \mathrm{a}$ \\
M4 & $7.89 \mathrm{a}$ & $1.98 \mathrm{a}$ & $1.53 \mathrm{a}$ & $60.52 \mathrm{~b}$ & $13.41 \mathrm{~b}$ & $4.23 \mathrm{a}$ & $2.39 \mathrm{~b}$ & $13.19 \mathrm{a}$ & $33.38 \mathrm{a}$ \\
DMS (0.05) & 0.56 & 0.13 & 0.08 & 2.28 & 0.8 & 0.28 & 0.16 & 0.86 & 2.39 \\
\hline
\end{tabular}

$\mathrm{RG}=$ rendimiento de grano; $\mathrm{AP}=$ altura de planta; $\mathrm{AM}=$ altura de mazorca; $\mathrm{FF}=$ floración femenina; $\mathrm{LM}=$ longitud de mazorca; $\mathrm{DM}=$ diámetro de mazorca; $\mathrm{DO}=$ diámetro de olote; $\mathrm{HM}=$ hileras por mazorca; $\mathrm{GH}=$ granos por hilera.

Para altura de planta (AP) las hembras con porte más alto fueron H6 (2.00 m) y H2 (1.99 m), este tipo de altura es idóneo para cosechas mecánicas debido a que pueden resistir el acame, las hembras presentaron en promedio una altura de mazorca (AM) favorable para las cosechas mecánicas fueron H3 (1.44 m) y H4 (1.43 m), en floración femenina los promedios en las siete hembras fluctuó entre los 74 a 76 días (d), y se consideran como de ciclo intermedio, lo que es una ventaja ya que los agricultores prefieren sembrar materiales de éste ciclo, en longitud de mazorca (LM) la H7 presentó el mayor tamaño con $14.88 \mathrm{~cm}$, mientras que la H5 quedo en segundo grupo estadístico con 14.31 $\mathrm{cm}$, el resto de las hembras se concentraron en diferentes grupos, en diámetro de mazorca (DM) las hembras H1, H6 y $\mathrm{H} 3$ presentaron promedios de 4.66, 4.31 y $4.23 \mathrm{~cm}$ respectivamente; en hileras por mazorca $(\mathrm{HM})$ y granos por hilera $(\mathrm{GH})$, no hubo diferencias estadísticas. 
Para machos no hubo significancia estadística en (RG), (AP), (DM) y (HM); sin embargo, para altura de mazorca (AM), el M4 presentó un promedio de $1.53 \mathrm{~m}$ y M1 obtuvo el valor más bajo con $1.29 \mathrm{~m}$, para floración femenina, M4 fue más precoz con $60 \mathrm{~d}$, mientras que el resto de los machos un promedio de $80 \mathrm{~d}$, en longitud de mazorca y diámetro de olote no hubo diferencias marcadas, solo 1.2 y $0.23 \mathrm{~cm}$ fue el rango de diferencia en ambas variables, en granos por hilera el promedio más bajo se presentó en M2 con 30.38 y mayor promedio lo obtuvo M3 con 34.24.

Al conjuntar, por recombinación y selección algunas líneas contrastantes, podría resultar en patrones heteróticos definidos porque, según Dzib et al. (2011), los híbridos altamente rendidores lo fueron por la heterosis y también por factores hereditarios, como la interacción múltiple de genes de tipo aditivo. Esto muestra la conveniencia de conocer la importancia relativa de cada componente genético en la producción de híbridos. Las líneas H3 y H5 fueron las que intervinieron en las cruzas más sobresalientes por su rendimiento, seguidas de H6 y H7. El M2 fue la que apareció con mayor frecuencia en cruzas superiores, por lo que las líneas H6 y M2 serían indicadas para separar el resto de las líneas en dos grupos heteróticos, (Malacarne y San Vicente, 2003).

\section{Aptitud combinatoria general (ACG)}

Los mayores efectos de aptitud combinatoria general para RG y DM se encontraron en las hembras H4 y H2 (Cuadro 4); sin embargo, H5 y H1 obtuvieron valores inferiores y negativos, lo anterior sugiere que $\mathrm{H} 4$ y $\mathrm{H} 2$ contienen genes de efectos aditivos que se expresan favorablemente en el rendimiento de grano (Avila et al., 2009). Para AP, H2 y H3 presentaron mayores efectos en FF, H6 y $\mathrm{H} 4$ obtuvieron valores superiores, para LM las hembras $\mathrm{H} 1$ y $\mathrm{H} 3$ indicaron que fueron de mayor expresión con efectos positivos, sin embargo, para DO, HM y GH, H3 y H5 obtuvieron en promedio los máximos valores, todos con efectos positivos y también con los máximos valores per se registrados; es decir, en promedio estas poblaciones generaron las mejores combinaciones.

Cuadro 4. Valores estimados de aptitud combinatoria general (ACG) de líneas de maíz utilizadas como machos y hembras, en el diseño II de Carolina del Norte. Marín, Nuevo León.

\begin{tabular}{cccccccccc}
\hline Línea & $\begin{array}{c}\mathrm{RG} \\
\left(\mathrm{t} \mathrm{ha}^{-1}\right)\end{array}$ & $\begin{array}{c}\mathrm{AP} \\
(\mathrm{m})\end{array}$ & $\begin{array}{c}\mathrm{AM} \\
(\mathrm{m})\end{array}$ & $\begin{array}{c}\mathrm{FF} \\
(\mathrm{días})\end{array}$ & $\begin{array}{c}\mathrm{LM} \\
(\mathrm{cm})\end{array}$ & $\begin{array}{c}\mathrm{DM} \\
(\mathrm{cm})\end{array}$ & $\begin{array}{c}\mathrm{DO} \\
(\mathrm{cm})\end{array}$ & $\mathrm{HM}$ & $\mathrm{GH}$ \\
\hline H1 & -0.19 & -0.49 & 0.95 & 0.8 & 0.8 & 2.56 & -0.29 & -0.1 & 0.02 \\
$\mathrm{H} 2$ & 0.13 & 0.35 & 0.9 & -0.16 & -0.16 & 1.81 & 0.3 & 0.01 & 0 \\
H3 & -0.01 & 0.23 & 0.91 & 1 & 1 & 2.06 & 0.03 & 0.11 & 0.01 \\
H4 & 0.14 & 0.1 & 0.89 & -0.16 & -0.16 & 0.89 & 0.8 & 0.02 & 0.02 \\
H5 & -0.03 & -0.13 & 0.9 & -0.04 & -0.04 & -2.27 & 0.22 & 0.07 & 0.06 \\
H6 & 0.08 & -0.05 & 0.87 & -0.33 & -0.33 & -3.77 & -0.51 & -0.04 & -0.04 \\
H7 & 0.04 & -0.01 & 0.85 & 0.25 & 0.25 & -1.27 & -0.55 & -0.06 & -0.07 \\
M1 & 0.08 & 0.12 & 0.7 & -0.06 & 8.01 & 8.45 & 0.02 & 0.03 & 0.1 \\
M2 & 0.19 & 0.04 & 0.75 & -0.08 & 7.38 & 7.79 & -0.05 & 0.04 & -0.04 \\
M3 & -0.25 & -0.32 & 0.65 & 0.04 & -1.47 & -1.55 & -0.35 & -0.06 & 0 \\
M4 & 0.04 & 0.15 & 0.85 & 0.1 & -13.93 & -14.69 & 0.37 & -0.01 & -0.06 \\
\hline
\end{tabular}

$\mathrm{RG}=$ rendimiento de grano; $\mathrm{AP}=$ altura de planta; $\mathrm{AM}=$ altura de mazorca; $\mathrm{FF}=$ floración femenina; $\mathrm{LM}=$ longitud de mazorca; DM= diámetro de mazorca; DO= diámetro de olote; HM= hileras por mazorca; $\mathrm{GH}=$ granos por hilera. 
Estos resultados indican que dichas poblaciones tienen una alta contribución en la expresión de las características evaluadas en sus progenies, y que los efectos aditivos son los más importantes; por lo tanto, pueden incluirse en un programa de mejoramiento genético de maíz, para contribuir con alelos superiores (De la Cruz et al., 2005; Preciado et al., 2005; Guillén et al., 2009). Se observa que de acuerdo con los efectos de ACG registrados existen otras poblaciones con orígenes diversos que pueden aportar al mejoramiento genético del maíz y que hasta ahora se ha prestado poca atención a sus potenciales como fuentes para incrementar la base genética de los híbridos comerciales (Guillén et al., 2009).

Los machos que expresaron mayor aptitud combinatoria para RG y LM fueron M2 y M1, en AP, AM, FF, LM, DM, DO, HM y GH, M1, M3 y M4 obtuvieron valores superiores y positivos asociados entre estas variables de ACG, lo que indica que no todos los componentes del rendimiento de grano tienen comportamiento similar, sino que dependen de la constitución genética de cada línea; además, M4 mostró valores superiores en seis (60\%) de las características, lo cual puede ser ventajoso en el mejoramiento del rendimiento, como lo mencionan Wong et al. (2007).

En todos los caracteres antes señalados los efectos de ACG fueron mayores a los correspondientes a la ACE, aunque es importante señalar la presencia de genes dominantes, pero los efectos de genes aditivos contribuyen en mayor proporción que los efectos no aditivos en la expresión del fenotipo, lo cual coincide con Badawy (2013) para la variable rendimiento de grano. Sin embargo, estos resultados difieren con los publicados por Kanagarasu et al. (2010); Borghi et al. (2012) quienes encontraron que los efectos no aditivos fueron superiores a los aditivos para rendimiento de grano, diámetro de mazorca, longitud de mazorca y altura de planta.

\section{Aptitud combinatoria específica (ACE)}

En el Cuadro 5, se muestra el valor promedio y los efectos de ACE de los 28 híbridos, observándose que las cinco mejores combinaciones para RG (Cuadro 6) se presentaron en (H4*M2), (H6*M3), $(\mathrm{H} 2 * \mathrm{M} 3),(\mathrm{H} 5 * \mathrm{M} 4)$ y $(\mathrm{H} 6 * \mathrm{M} 4)$; sin embargo, el híbrido $(\mathrm{H} 1 * \mathrm{M} 2)$ resultó ser el que expresó menor aptitud con un valor negativo, donde el máximo valor fue de $1.17 \mathrm{t} \mathrm{ha}^{-1}$, que correspondió al primer cruzamiento enlistado, lo que se esperaba ya que las líneas progenitoras de H4xM2 expresaron los mayores efectos de ACG (Cuadro 5), lo anterior, puede deberse a la suma de los efectos aditivos de los genes de los progenitores o a un efecto alto positivo de ACE, este resultado sugiere que la manifestación de alto rendimiento en la cruza es debida a la acción génica de efectos aditivos de las líneas, como aseveran Springer y Stupar (2007). Se detectó que, en los cruzamientos con ACE de mayor magnitud, combinaron poblaciones de origen geográfico contrastado en el área de distribución de Nuevo León y la dimensión de los efectos registrados fue debido a la distancia que existe entre ambas poblaciones; H4 pertenece al municipio de Pesquería y M2 al de Los Ramones.

En AP, los mejores cinco híbridos que expresaron mayores efectos de ACE fueron: H2xM4, $\mathrm{H} 3 \times \mathrm{M} 1, \mathrm{H} 4 * \mathrm{M} 2$, H6*M1 y H5*M2. Las poblaciones pertenecientes a las variantes de los híbridos tuvieron influencia importante sobre la expresión de los cruzamientos sobresalientes para esta variable, donde dichos cruzamientos en su mayoría presentaron tanto valores promedio como efectos de ACE altos, en AM las mejores cruzas fueron (H4*M4), (H4*M2), (H5*M2), (H2*M1) y (H7*M4). Para FF y LM, las cruzas simples $\mathrm{H} 2 * \mathrm{M} 3$ y H3*M3, expresaron mayores efectos de ACE; sin embargo, en DM, DO, HM y GH, fueron $\mathrm{H} 1 * \mathrm{M} 3, \mathrm{H} 1 * \mathrm{M} 1$ y $\mathrm{H} 5 * \mathrm{M} 1$. 
Cuadro 5. Valores estimados de aptitud combinatoria específica (ACE) de 20 cruzas con mayor rendimiento y sus componentes agronómicos generadas por el diseño II de Carolina del Norte. Marín, Nuevo León.

\begin{tabular}{lccccccccc}
\hline Cruza & $\begin{array}{c}\text { RG } \\
\left(\mathrm{t} \mathrm{ha}^{-1}\right)\end{array}$ & $\begin{array}{c}\text { AP } \\
(\mathrm{m})\end{array}$ & $\begin{array}{c}\text { AM } \\
(\mathrm{m})\end{array}$ & $\begin{array}{c}\text { FF } \\
(\mathrm{días})\end{array}$ & $\begin{array}{c}\text { LM } \\
(\mathrm{cm})\end{array}$ & $\begin{array}{c}\text { DM } \\
(\mathrm{cm})\end{array}$ & $\begin{array}{c}\text { DO } \\
(\mathrm{cm})\end{array}$ & HM & GH \\
\hline H4xM2 & 1.17 & 0.65 & 0.7 & 1.41 & 0.3 & 0.81 & 0.18 & -0.03 & 0.41 \\
H6xM3 & 0.84 & -0.01 & -0.29 & -13.47 & -11.37 & -0.98 & 0 & 0.09 & 0.39 \\
H2xM3 & 0.58 & -0.36 & 0.32 & 11.01 & 10.71 & -0.08 & -0.24 & -0.04 & -0.32 \\
H5xM4 & 0.58 & -0.64 & 0.25 & -3.04 & -0.39 & -0.46 & 0.1 & -0.01 & -0.55 \\
H6xM4 & 0.43 & 0.11 & 0.34 & -0.68 & 3.77 & 0.49 & 0 & -0.01 & -0.18 \\
H2xM2 & 0.4 & -0.26 & 0.25 & -1.24 & -3.62 & -0.25 & 0.01 & -0.12 & 0.83 \\
H1xM3 & 0.32 & 0.19 & -0.19 & 8.94 & 7.63 & 2.26 & 0.28 & 0 & 0.39 \\
H3xM1 & 0.14 & 0.66 & -0.23 & -0.62 & -3.2 & 1.88 & -0.02 & -0.04 & -0.13 \\
H5xM2 & 0.13 & 0.38 & 0.49 & 2 & 4.13 & 0.12 & 0.19 & 0.15 & -0.67 \\
H4xM1 & 0.06 & -0.63 & -0.44 & -2.1 & -3.7 & -1.78 & -0.12 & 0.13 & -0.54 \\
H7xM4 & 0.03 & -0.1 & 0.46 & 1.98 & 4.27 & 2.11 & 0.01 & -0.04 & 0.15 \\
H5xM1 & 0.03 & 0.18 & 0.15 & 3.51 & 5.8 & 0.65 & 0.03 & -0.06 & 1.04 \\
H1xM4 & 0.02 & 0.3 & 0.14 & 1.68 & 0.11 & -1.04 & -0.22 & -0.13 & 0.15 \\
H3xM3 & 0.02 & -0.2 & -0.47 & 11.01 & 10.46 & -1.73 & 0.12 & -0.02 & -0.32 \\
H3xM2 & -0.05 & -0.29 & 0.15 & 1.12 & -1.2 & 0.39 & -0.05 & 0 & 0.16 \\
H2xM1 & -0.08 & -0.17 & 0.46 & -1.51 & -3.95 & 0.04 & 0.03 & 0.03 & -0.79 \\
H4xM4 & -0.08 & -0.29 & 0.7 & 1.39 & 1.44 & -0.86 & -0.04 & 0 & -0.14 \\
H4xM3 & -0.09 & 0.26 & -0.74 & 2.46 & 1.96 & 1.83 & -0.02 & -0.09 & 0.27 \\
H7xM3 & -0.1 & 0.03 & -0.49 & -9.93 & -9.87 & -0.98 & 0.19 & 0.16 & -0.61 \\
H7xM1 & -0.12 & 0.27 & -0.13 & 2.03 & 3.13 & -1.23 & -0.1 & -0.12 & 0.42 \\
\hline
\end{tabular}

$\mathrm{RG}=$ rendimiento de grano; $\mathrm{AP}=$ altura de planta; $\mathrm{AM}=$ altura de mazorca; $\mathrm{FF}=$ floración femenina; $\mathrm{LM}=$ longitud de mazorca; $\mathrm{DM}=$ diámetro de mazorca; $\mathrm{DO}=$ diámetro de olote; $\mathrm{HM}=$ hileras por mazorca; $\mathrm{GH}=$ granos por hilera.

Por otra parte, de los ocho progenitores que intervienen en las cruzas con mayores efectos de ACE, la $\mathrm{H} 1$ y M1 presentaron altos efectos de ACG (Cuadro 5), lo cual indica que sus progenitores son adecuados para formar híbridos intervarietales con alto potencial de rendimiento, o para derivar líneas que al cruzarse tengan buena combinación entre ellas. Al respecto, se espera que las cruzas con mayor ACE resulten de cruzar al menos una población con alta ACG (Legesse et al., 2009; Escorcia et al., 2010).

Los progenitores $\mathrm{H} 4, \mathrm{H} 2$, M1 y M2 fueron los que más rindieron (de 7.3 a $8.1 \mathrm{t} \mathrm{ha}^{-1}$ ), presentaron un porte de planta (de $1.8 \mathrm{~A} 1.9 \mathrm{~m}$ ) y mazorca (de 1.2 a $1.4 \mathrm{~m}$ ), de un ciclo intermedio (de 76 a 81 días a FF), pero de mayor longitud de mazorca $(13$ a $14.3 \mathrm{~cm})$, mayor número de hileras por mazorca (12.6 a 13) y mayor número de granos por hilera (30.3 a 33) con respecto al resto de las líneas. 
Esto indica que en la herencia de las cruzas participaron genes no aditivos. La acción no aditiva de los genes fue reducida entre las cruzas de los progenitores, la participación de genes no aditivos en las mejores cruzas puede deberse a que los progenitores tienen amplia variación genética y a mayores efectos aditivos como se corrobora con los altos valores de ACG encontrados en las variables RG, AP, AM, LM, DM, DO, HM y GH.

Estos resultados coinciden con lo reportado por (Dzib et al., 2011), quienes reportaron que los genes no aditivos contribuyeron más en la expresión de la longitud y diámetro de mazorca, número de hileras para la obtención del mayor rendimiento de grano. Las cruzas con mayores valores de ACE pueden considerarse como prospectos en programas de mejoramiento genético para formar híbridos y para introducir variación genética en programas de selección reciproca recurrente. Los valores fueron pequeños comparados con los que obtuvieron Reyes et al. (2004); Escorcia et al. (2010), pero similares a los de Legeese et al. (2009).

\section{Componentes genéticos}

Al estimar los componentes genéticos (Cuadro 6), en rendimiento de grano y longitud de mazorca, las varianzas de híbridos, machos, hembras e interacción machos por hembras se observó valores elevados; sin embrago, en la varianza ambiental las variables como RG, AP, LM, DO y GH fueron superiores con respecto a la varianza aditiva y de dominancia, por lo que la varianza ambiental influyó en mayor proporción a la expresión de la varianza fenotípica respecto a estos caracteres. Para el caso de la heredabilidad en sentido amplio, el RG, AM y HM sobresalieron, con excepción en la LM, AM y FF para heredabilidad en sentido estrecho.

Cuadro 6. Valores estimados de los componentes de varianza genéticos para rendimiento de grano y sus componentes agronómicos. Marín, Nuevo León.

\begin{tabular}{cccccccccc}
\hline Componente & $\delta^{2}$ Híbridos & $\delta^{2} \mathrm{M}$ & $\delta^{2} \mathrm{H}$ & $\delta^{2} \mathrm{MxH}$ & $\delta^{2} \mathrm{~A}$ & $\delta^{2} \mathrm{D}$ & $\delta^{2} \mathrm{~F}$ & $\mathrm{H}^{2}$ & $\mathrm{~h}^{2}$ \\
\hline $\mathrm{RG}$ & 11.36 & 15.87 & 7.2 & 8.45 & 3.65 & 5.71 & 13.49 & 87.14 & 10.1 \\
$\mathrm{AP}$ & 0.31 & 4.51 & 0.87 & 0.61 & 0.2 & 0.7 & 1.26 & 65.15 & 31.05 \\
$\mathrm{AM}$ & 0.87 & 0.6 & 0.52 & 0.75 & 0.35 & 1.15 & 1.87 & 86.91 & 46.25 \\
$\mathrm{FF}$ & 1.1 & 4.38 & 3.7 & 1.89 & 1.49 & 3.2 & 5.52 & 63.38 & 34.7 \\
$\mathrm{LM}$ & 23.74 & 19.69 & 10.15 & 43.87 & 1.98 & 15.46 & 4.9 & 55.1 & 52.15 \\
$\mathrm{DM}$ & 1.65 & 5.87 & 8.1 & 6.7 & 1.41 & 3.93 & 6.86 & 65.82 & 28.14 \\
$\mathrm{DO}$ & 1.36 & 0.97 & 5.05 & 5.62 & 3.46 & 7.11 & 0.95 & 63.21 & 29.34 \\
$\mathrm{HM}$ & 8.37 & 15.1 & 3.84 & 2.79 & 1.15 & 2.1 & 4.28 & 73.14 & 30.16 \\
$\mathrm{GH}$ & 1.7 & 3.68 & 8.6 & 4.21 & 3.46 & 3.98 & 1.5 & 63.16 & 29.37 \\
\hline
\end{tabular}

$\mathrm{RG}=$ rendimiento de grano; $\mathrm{AP}=$ altura de planta; $\mathrm{AM}=$ altura de mazorca; $\mathrm{FF}=$ floración femenina; $\mathrm{LM}=$ longitud de mazorca; $\mathrm{DM}=$ diámetro de mazorca; $\mathrm{DO}=$ diámetro de olote; $\mathrm{HM}=$ hileras por mazorca; $\mathrm{GH}=$ granos por hilera. $\delta^{2} \mathrm{M}=$ varianza de machos; $\delta^{2} \mathrm{H}=$ varianza de hembras; $\delta^{2} \mathrm{~A}=$ varianza aditiva; $\delta^{2} \mathrm{D}=$ varianza de dominancia; $\delta^{2} \mathrm{~F}=$ varianza fenotípica y $\mathrm{h}^{2}=$ heredabilidad.

Estos resultados se atribuyen a la diversidad de la constitución genética de las líneas progenitoras, que al aparearse forman individuos de tipo heterocigótico, que reducen los efectos aditivos. Por tal razón, es de suponerse que todas las variaciones están estimadas con base en el comportamiento 
genético determinado como varianza de dominancia, lo cual es de esperarse puesto que existe una mayor expresión heterótica en los híbridos por efecto de la ACE de la mayoría de las líneas en RG, lo que da como resultado un valor más alto de heredabilidad en el sentido amplio por efecto de la varianza de dominancia (Badawy, 2013). Hallauer et al. (2010) señalaron que otra de las posibles razones de una menor varianza aditiva, es el origen de los progenitores que forman los híbridos, ya que cuando provienen de una sola población o de poblaciones emparentadas, hay alta probabilidad de que sobresalga este tipo de acción génica, como es el caso del parentesco entre las líneas M2 (Población 19 de Liebre, precoz tolerante a sequía) y la H4 (Población 11 de Pinto Amarillo, de ciclo intermedio de amplia adaptabilidad) utilizadas en este trabajo.

La mayor contribución se encontró en la varianza ambiental $\left(\sigma^{2} \mathrm{~F}\right)$, por lo que se sugiere formar híbridos superiores en rendimiento y sus componentes, con la selección de líneas que se complementen en sus caracteres, así como incrementar las líneas sobresalientes en ACG y ACE, analizar y seleccionar para rendimiento de grano, con la intención de liberar nuevos híbridos (Preciado et al., 2005). Por lo tanto, se sugiere formar variedades sintéticas heteróticas (con 8 a 10 líneas), para lo cual se seleccionan líneas que se complementen en sus caracteres y que formen poblaciones contrastantes para recombinar y extraer nuevas líneas para formar híbridos superiores en rendimiento, en esquemas de selección recíproca recurrente (Hallauer et al., 2010) los valores de heredabilidad en sentido estricto $\left(\mathrm{h}^{2}\right)$ para el DM, DO y GH fue de 28.14, 29.34 y 29.37\%, respectivamente, coinciden con lo reportado por Molina (1992) quien los identificó como caracteres cuantitativos de heredabilidad intermedia. La $\mathrm{h}^{2}$ del RG también coincidió con la clasificación del citado autor (carácter con heredabilidad baja).

La heredabilidad se refiere a la capacidad que tienen los caracteres para transmitirse de generación en generación; es decir, que ésta se pueda considerar como el grado de parecido entre los individuos de una generación y la siguiente, por lo que se considera estos serían los caracteres que se trataría de recombinar y seleccionar. Los componentes (LM, DO, DM e HM) que más influyeron en el rendimiento de grano, presentaron $\mathrm{h}^{2}$ altas (Molina, 1992). Esto indica que están estrechamente asociados con el rendimiento de grano, lo cual se considera alto ya que son caracteres cuantitativos, excepto para las variables de heredabilidad negativa cuyo valor parece lógico al ser más de caracteres cualitativos. Estos efectos también pueden deberse a que un mismo grupo de genes no controlan todas las respuestas de los componentes genéticos como lo señalan Springer y Stupar (2007) o factores hereditarios como la aditividad, la dominancia y la sobredominancia, según Lippman y Zamir (2007).

\section{Conclusiones}

El análisis de los resultados de las cruzas sugiere que las líneas con mayor ACG obtuvieron el mejor rendimiento de grano, lo cual permitió definir el aprovechamiento de los progenitores a través del sistema de hibridación como el más indicado para el programa de mejoramiento genético, ya que sus progenitores son adecuados para formar híbridos con alto potencial de rendimiento. Las líneas que obtuvieron mayor ACG se aprovecharán para la producción de semilla híbrida. Las líneas y las cruzas con rendimiento superior se aprovecharán para la producción de grano en la localidad de evaluación. Los valores bajos de la varianza aditiva se obtuvieron en la mayoría de las variables, a excepción de granos por hilera, características que pueden mejorarse a largo plazo. Las estimaciones de la heredabilidad en sentido amplio variaron $63.16 \%$ a $87.14 \%$. 


\section{Literatura citada}

Avila, P. M. A.; Rodríguez, H. S. A.; Vázquez, B. M. E.; Borrego, E. F.; Lozano, R. A. J. y López B. A. 2009. Aptitud combinatoria y efectos recíprocos en líneas endogámicas de maíz de valles altos del centro de México. Agric. Téc. Méx. 35(3):285-293.

Badawy, M. E. M. 2013. Heterosis and combining ability in maize using diallel crossed among seven new inbred lines. Asian J. Crop Sci. 5(1):1-13.

Borghi, M. L.; Ibañez, A. M.; Bonamico, C. N.; Kandus, V. M.; Gomar, A. D.; Guillin, A. E.; Salermo, C. J. and Renzo A. D. M. 2012. Combining ability of Flint corn inbred lines: Mal del Rio Cuarto Disease tolerance and grain yield. J. Exp. Bot. 81(1):123-131.

Comstock, R. E. and Robinson F. H. 1948. The components of genetic variance in populations of biparental progenies and their use in estimating the average degree of dominance. Biometrics. 4(4):254-266.

De la Cruz, L. E.; Rodríguez, H. S.; Estrada, M. A. B. y Mendoza D. P. J. 2005. Análisis dialélico de líneas de maíz QPM para características forrajeras. Univ. Cienc. 21(41):19-26.

De la Rosa, L. A.; De León, C. H.; Rincón, S. F. y Martínez, Z. G. 2006. Efectos genéticos. heterosis y diversidad genética entre híbridos comerciales de maíz adaptados al Bajío Mexicano. Rev. Fitotec. Mex. 29(3):247-254.

Dzib, A. L. A.; Segura, C. C. J.; Ortega, R. P. y Latournerie M. L. 2011. Cruzas dialélicas entre poblaciones nativas de Yucatán y poblaciones mejoradas. Trop. Subtrop. Agroecosys. 14(1):119-127.

Escorcia, G. N.; Molina, G. J. D.; Castillo, G. F. y Mejía, C. J. 2010. Rendimiento, heterosis y depresión endogámica de cruzas simples de maíz. Rev. Fitotec. Mex. 33(3):271-279.

Fan, X. M.; Chen, H. M.; Tan, J.; Xu, C. X.; Zhang, Y. D.; Luo, L. M.; Huang, Y. X. and Kang, M. S. 2008. Combining abilities for yield and yield components in maize. Maydica. 53(1):39-46.

Guillén, D. C. P.; De la Cruz, E. L.; Castañón, N. G.; Osorio, R. O.; Brito, P. M. N.; Lozano, R. A. D. y López, N. U. 2009. Aptitud combinatoria general y específica de germoplasma tropical de maíz. Trop. Subtrop. Agroecosys. 10(1):101-107.

Hallauer, A. R.; Carena, M. and Miranda, F. J. B. 2010. Quantitative genetics in maize breeding. Springer-Verlag. New York Inc. 664 p.

INEGI. 2013. Instituto Nacional de Estadística, Geografía e Informática. Modificaciones al sistema de clasificación climática de Köppen para adaptarlos a las condiciones de la República Mexicana. Universidad Nacional Autónoma de México (UNAM). México, DF. 246 p.

Kanagarasu, S.; Nallathambi, G. G. and Ganesan N. K. 2010. Combining ability analysis for yield and its components traits in maize (Zea mays L.). Electron. J. Plant Breed. 1(4):915-920.

Kang, S.M.; Kushairi, A. D.; Zhang, Y. and Magari, R. 1999. Combining ability for rind puncture resistance in maize. Crop Sci. 39(2):368-371.

Legesse, B. W.; Pixley, K. V. and Botha, A. M. 2009. Combining ability and heterotic grouping of highland transition maize inbred lines. Maydica. 54(1):1-9.

Lippman, Z. B. and Zamir, D. 2007. Heterosis: revisiting the magic. Trends in Genetics 23(2):60-66.

Louette, D. and Smale, M. 1996. Genetic diversity and maize seed management in a traditional Mexican community: implications for in situ conservation of maize. NRG Paper 96-03. Centro Internacional de Maíz y Trigo (CIMMYT). México, DF. 21 p. 
Malacarne, M. F. and San Vicente, G. F. M. 2003. Patrones heteróticos de líneas tropicales blancas de maíz. Agron. Trop. 53(4):32-40.

Polanco, F. 2008. Bases para una política de I\&d e innovación de la cadena de valor del maíz. Foro Consultivo Científico y Tecnológico. AC. México, DF. 244 p.

Preciado, O. E. R.; Terrón, I. A. D.; Gómez M. N. O. y Robledo, G. E. I. 2005. Componentes genéticos en poblaciones heteróticamente contrastantes de maíz de origen tropical y subtropical. Agron. Mesoam. 16(2):145-151.

Reyes, L. D.; Molina, D. G. J.; Oropeza, A. R. M. y Moreno, C. P. E. 2004. Cruzas dialélicas entre líneas autofecundadas de maíz derivadas de la raza tuxpeño. Rev. Fitotec. Mex. 27(1):49-56.

Rodríguez, P. G.; Zavala, G. F. G.; Ojeda, Z. C.; Gutiérrez, D. A.; Treviño, R. J. E. y Rincón S. F. 2012. Diversidad de maíces criollos de Nuevo León. México. Mediante AFLP y caracteres morfológicos. Agron. Mesoam. 23(2):29-39.

Soengas, P.; Ordaz, B.; Malvar, A. R.; Revilla, P. and Ordaz, A. 2003. Heterotic patterns among. Flint maize populations. Crop Sci. 43(3):844-849.

Sprague, G. F. and Tatum, A. L. 1942. General versus specific combining ability in single crosses of corn. J. Am. Soc. Agron. 34(10):923-932.

Springer, N. M. and Stupar, M. R. 2007. Allelic variation and heterosis in maize: how do two halves make more than a whole? Genome Res. 17(3):264-275.

Wong, R. R.; Gutiérrez, R. E.; Palomo, G. A.; Rodríguez, H. S.; Córdova, O. H.; Espinoza, B. A. y Lozano, G. J. J. 2007. Aptitud combinatoria de componentes de rendimiento en líneas de maíz para grano en la comarca lagunera. México. Rev. Fitotec. Mex. 30(2):181-189. 